\title{
I 032 Prognostic impact of contrast-enhanced CMR early after acute ST-segment elevation myocardial infarction (STEMI) in a regional STEMI network
}

\author{
Oliver Bruder*1, Peter Hunold ${ }^{2}$, Christoph Jensen ${ }^{1}$, Markus Jochims ${ }^{1}$, \\ Georg V Sabin ${ }^{1}$ and Joerg Barkhausen ${ }^{2}$
}

\author{
Address: ${ }^{1}$ Department of Cardiology and Angiology, Elisabeth Hospital Essen, Essen, Germany and ${ }^{2}$ Department of Diagnostic and Interventional \\ Radiology and Neuroradiology University Hospital Essen, University of Duisburg-Essen, Essen, Germany \\ * Corresponding author
}

from I Ith Annual SCMR Scientific Sessions

Los Angeles, CA, USA. I-3 February 2008

Published: 22 October 2008

Journal of Cardiovascular Magnetic Resonance 2008, I0(Suppl I):AI57 doi:I0.I I86/I532-429X-10-SI-AI57

This abstract is available from: http://jcmr-online.com/content/I0/SI/AI57

(C) 2008 Bruder et al; licensee BioMed Central Ltd.

\section{Introduction}

In acute ST-segment elevation myocardial infarction (STEMI), rapid restoration of epicardial coronary blood flow and myocardial perfusion limits infarct size and improves survival. Primary percutaneous coronary intervention (PCI) is superior to systemic fibrinolysis when instantly performed by experienced operators. The "Herzinfarktverbund Essen" (HIVE) is an urban STEMI network supporting direct patient transfer for primary PCI to 4 PCI centers covering a city area of 600.000 inhabitants. Integrated health care is an optional part of the HIVE allowing for reimbursement of medical innovations such as the evaluation of infarct size and the presence and extent of microvascular obstruction by contrast-enhanced CMR.

\section{Objectives}

Aim of the study was to assess the prognostic impact of contrast-enhanced CMR in a patient cohort of a regional STEMI network.

\section{Methods}

Within the first year (09/04 to 08/05) of the HIVE registry 489 patients with acute myocardial infarction were treated in the 4 primary PCI centers. In one of the centers, including 143 patients, early CMR imaging using a standardized MR protocol for infarct quantification was performed whenever possible. Patients with hemodynamic instabil- ity, emergency $C A B G$, resuscitation or death prior to CMR, claustrophobia, and other general contraindications to MRI had to excluded leaving 67 patients (54 male; mean age, $61 \pm 12$ years) for the final evaluation.

CMR was performed $4.5 \pm 2.5$ days after admission on a 1.5 Tesla MR Scanner (Avanto, Siemens Medical Solutions, Erlangen, Germany) including steady-state free precession (SSFP) cine imaging for LV function and single shot inversion-recovery SSFP imaging for delayed enhancement (DE) and no-reflow (NR) evaluation following injection of $0.2 \mathrm{mmol} / \mathrm{kg}$ BW gadodiamide (Omniscan, GE Healthcare Buchler, Munich, Germany). $\mathrm{LV}$ volumes and myocardial mass were calculated from planimetry of contiguous short axis slices and summation of discs. NR volumes were calculated from single-shot short axis stacks taken within the first minute following gadodiamide infusion by manual planimetry and summation of discs. DE volumes were calculated from the inversion-recovery SSFP images taken at least 10 minutes after contrast administration.

1-year-follow-up data (telephone interview) for major adverse cardiac events (MACE: cardiac death, myocardial infarction, and re-hospitalization for congestive heart failure, angina pectoris, or revascularization) were available from all patients. 


\section{Results}

DE as a measure of infarct size was $9 \pm 7 \%$ ( 0 to $33 \%$ ) of $\mathrm{LV}$ mass, and mean volume of microvascular obstruction was $2 \pm 3 \%$ (range 0 to $17 \%$ ). Microvascular obstruction was present in $61 \%$ of patients.

Infarct localization was anterior in 37 patients (infarct size, $10 \pm 9 \%$ of LV mass; microvascular obstruction, $3 \pm$ $4 \%$ of LV mass; LVEF, $43 \pm 13 \%$; LVEDV, $143 \pm 37 \mathrm{ml}$; LVESV, $80 \pm 27 \mathrm{ml})$ with NR in 22 of $37(60 \%)$, and inferior in 30 cases (DE, $8 \pm 6 \%, \mathrm{NR}, 1 \pm 2 \%$; LVEF, $52 \pm 15 \%$; LVEDV, $146 \pm 52 \mathrm{ml}$; LVESV, $75 \pm 55 \mathrm{ml}$ ) with NR in 19 of $30(63 \%)$ patients.

16 MACE (1 cardiac death, 1 myocardial infarction, and 14 re-hospitalizations for congestive heart failure or unstable angina pectoris with PCI in 6 cases) occurred within the follow-up period of $430 \pm 63$ days.

Patients with MACE had larger infarcts $(14 \pm 10 \%$ vs. $8 \pm$ $6 \%$ DE), lower LVEF ( $44 \pm 17 \%$ vs. $48 \pm 14 \%)$ and larger NR ( $3 \pm 5 \%$ vs $2 \pm 3 \%)$. Using a stepwise logistic regression model, only NR $>0.5 \%$ of LV mass was independently related to outcome $(\mathrm{OR}=3.9$, CI 1.1-13.9).

\section{Conclusion}

No-reflow as a correlate of microvascular obstruction remains independently related to prognosis in patients with acute myocardial infarction treated by PCI. 


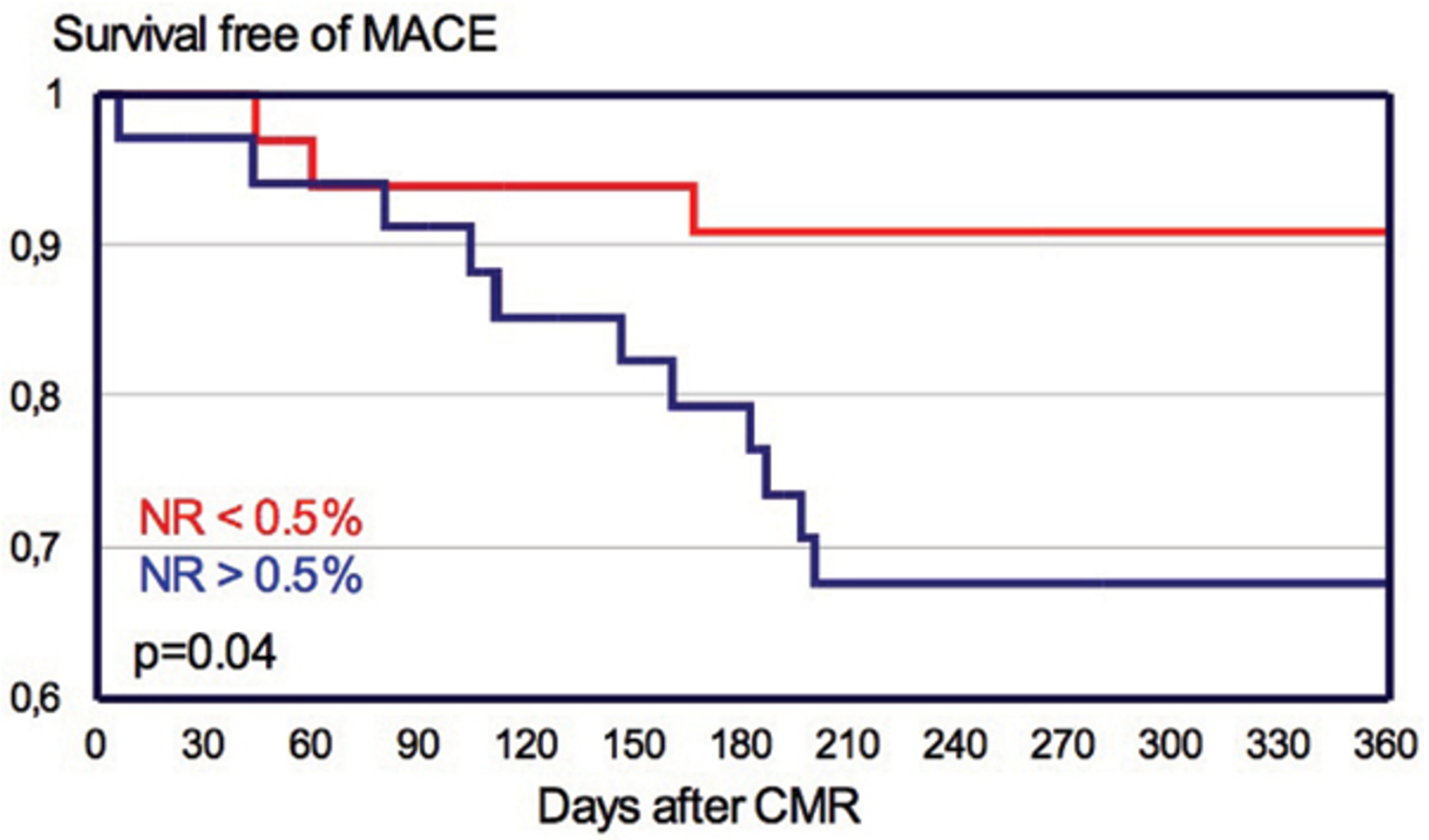

Figure I

Kaplan-Meier Curve for Event-free Survival.

Publish with Bio Med Central and every scientist can read your work free of charge

"BioMed Central will be the most significant development for disseminating the results of biomedical research in our lifetime."

Sir Paul Nurse, Cancer Research UK

Your research papers will be:

- available free of charge to the entire biomedical community

- peer reviewed and published immediately upon acceptance

- cited in PubMed and archived on PubMed Central

- yours - you keep the copyright
BioMedcentral 